TRENDS IN FATAL AND NONFATAL INJURIES

AMONG OLDER AMERICANS, 2004-2015

D. $\mathrm{Xu}^{1}, \mathrm{~J}$. Drew ${ }^{2}, 1$. Purdue University, 2. University of

Minnesota

The objectives were (1) to provide annual estimates of fatal and nonfatal injury incidence rates for older adults for the 2004-2015 period, (2) to determine whether observed incidence trends differ by characteristics of the injury: fatal or nonfatal, due to a fall or another cause, and whether the nonfatal injury was minor or serious. We used data covering the time period from three leading sources of information about injuries: National Vital Statistics System (NVSS), National Electronic Injury Surveillance System-All Injury Program (NEISS-AIP), and National Health Interview Survey (NHIS). Our results showed a clear increase in the fatal injury incidence rate among older adults, mainly attributable to fallrelated death. Estimates using data from the NEISS-AIP and NHIS gave substantively consistent results for trends in the overall rate of nonfatal injury incidence, which significantly increased over the past decade. Fall-related nonfatal injuries, like fall-related fatal injuries, increased considerably over the past decade. In contrast, the trend in other-causerelated injury incidence was fairly stable. Further analyses indicate that the observed increase in nonfatal injuries was dominated by an increase in minor injuries that were treated in a doctor's office, or via a call to a medical professional. Our findings suggest that efforts to prevent injuries, particularly fall-related injuries, should continue to be priorities. Extra efforts also need to focus on nonfatal injuries where treatment was not sought in an ER or hospital among older adults, which have long-lasting impacts on health and medical care utilization and are often overlooked in intervention programs.

\section{WHEN FUNCTIONAL IMPAIRMENT DEVELOPS EARLY: PERSPECTIVES FROM MIDDLE-AGED ADULTS}

E. Xu ${ }^{1}$, M. Barrientos ${ }^{1}$, F. Nicosia ${ }^{2}$, M. Steinman ${ }^{1}$, M. Spar ${ }^{3}$, L. Karliner ${ }^{1}$, R. Brown ${ }^{1}, 1$. University of California, San Francisco, 2. San Francisco VA Medical Center, San Francisco, CA, 3. University of California, San Francisco, San Francisco VA Medical Center

Difficulty performing basic activities of daily living (ADLs) is often seen as a problem affecting adults aged 65 and older, especially the oldest old. However, such difficulties also commonly develop among middle-aged adults, and these difficulties can persist and worsen over time. Yet little is known about the experiences and needs of individuals who develop ADL difficulty in middle age. We conducted semi-structured interviews with 40 patients ages 50-64 recruited from four primary care clinics in San Francisco. Patients were eligible if they spoke English or Spanish and were identified by their primary care providers as having developed difficulty performing one or more ADLs during middle age. Interviews focused on patients' daily life, needs, and experiences with the healthcare system. We analyzed transcripts iteratively using a thematic approach. Interviews revealed three main themes: 1) the importance of - and challenges to - developing "systems" to manage ADL impairments, including services, supports, and environmental adaptations; 2) psychosocial challenges related to reconciling ones' identity before and after developing ADL impairment; and 3) difficulty in having ones' needs met within the healthcare system due to its focus on individual diseases and diagnostic tests rather than function and quality of life. Middle-aged adults who develop functional impairment identified several key unmet needs, including psychosocial support and a clinical focus on function. Understanding and addressing these needs will be necessary to deliver optimal care to this population.

\section{SESSION 1780 (POSTER)}

\section{END-OF-LIFE ISSUES}

\section{A SYSTEMATIC REVIEW OF USING THE PALLIATIVE PERFORMANCE SCALE TO PREDICT SURVIVAL FOR OLDER ADULTS AT THE END-OF-LIFE}

D. Baik ${ }^{1}$, D. Russell ${ }^{2}$, L. Jordan 3 , F. Dooley ${ }^{3}$, K. Bowles ${ }^{4}$, R. Masterson Creber $^{5}, 1$. Columbia University, 2. Appalachian State University, 3. Visiting Nurse Service of New York, 4. University of Pennsylvania School of Nursing, 5. Columbia University School of Nursing

Older adults with terminal illness face many end-oflife care challenges. Specifically, knowing how much time remains is essential for closure for personal and family matters. The Palliative Performance Scale (PPS) has been widely used for survival prediction among patients with cancer; however, few studies have reviewed PPS scores in heterogeneous hospice populations across multiple care settings. The purpose of this systematic literature review was to determine how the PPS tool has been used to predict survival for older adults at the end-of-life. This review followed the Preferred Reporting Items for Systematic Reviews and Meta-Analyses guidelines and electronic databases (PubMed, Embase, Cochrane Library) were searched for all published literature from 2008 to 2017 . We analyzed study characteristics, the PPS score on admission, and primary outcomes, and explored differences in survival estimates by diagnosis. Seventeen studies were included in this review (9 with cancer and 8 with mixed diagnoses). The primary findings from this review were that the PPS predicted length of survival across all studies. In this review, survival estimates ranged from 1 to 3 days for older adults with PPS scores of $10 \%$ compared with a range of 5 to 36 days for those with scores of $30 \%$. The findings confirm that the PPS has been used more frequently to estimate survival in patients with cancer and that there are opportunities to apply the tool to patients with different end-of-life diagnoses.

\section{AN ADVANCE CARE PLANNING VIDEO DECISION SUPPORT TOOL FOR NURSING HOME RESIDENTS WITH ADVANCED DEMENTIA: A CLUSTER RCT}

S. Mitchell ${ }^{1}$, M. Shaffer ${ }^{2}$, S. Cohen ${ }^{3}$, L. Hanson ${ }^{4}$, D. Habtemariam ${ }^{3}$, A. Volandes ${ }^{5}, 1$. Harvard Medical School, 2. University of Washington, 3. Hebrew SeniorLife, 4. University of North Carolina School of Medicine, 5. Massachusetts General Hospital

Background: Better advance care planning (ACP) can help promote goal-directed care in advanced dementia. Methods: Educational Video to Improve Nursing home Care in Endstage dementia was a cluster randomized clinical trial conducted 2013-2017 in 64 Boston-area nursing homes (32 